\title{
Erratum to: Revisiting phylogenetic signal; strong or negligible impacts of polytomies and branch length information?
}

\author{
Rafael Molina-Venegas and Miguel Á. Rodríguez
}

\section{Erratum}

The original version of this manuscript [1] contained a mistake regarding the definition of Brownian motion model of evolution. This mistake does not affect the results of the manuscript, but it should be clarified.

Page 4. Second paragraph reads:

"Briefly, a BM model on a phylogeny describes purely neutral (random) evolution of a trait with variance proportional to the square root of branch lengths".

and it should read as follows:

"Briefly, a BM model on a phylogeny describes purely neutral (random) evolution of a trait with variance directly proportional to the branch lengths".

Also, there are two typos in the text:

"steaminess" should be "steminess" (third paragraph in page 5)

"Graphen" should be "Grafen" (fifth paragraph in page 8 and Conclusions)

\section{Acknowledgements}

We thank Dr. Simon Blomberg for pointing out the mistake.

Received: 27 March 2017 Accepted: 30 March 2017

Published online: 18 May 2017

\footnotetext{
References

1. Molina-Venegas R, Rodríguez, MA. Revisiting phylogenetic signal; strong or negligible impacts of polytomies and branch length information? BMC Evol Biol 10.1186/s12862-017-0898-y
}

\footnotetext{
* Correspondence: rmolina@us.es

Departamento de Ciencias de la Vida, Universidad de Alcalá, 28805 Alcalá de Henares, Madrid, Spain
} 